\title{
MAXIMAL ORDERS AND REFLEXIVE MODULES
}

BY

\section{J. H. COZZENS}

Dedicated to the memory of my mother

ABSTRACT. If $R$ is a maximal two-sided order in a semisimple ring and $M_{R}$ is a finite dimensional torsionless faithful $R$-module, we show that $m=$ End $_{R} M^{*}$ is a maximal order. As a consequence, we obtain the equivalence of the following when $M_{R}$ is a generator:

1. $M$ is $R$-reflexive.

2. $k=$ End $M_{R}$ is a maximal order.

3. $k=$ End $_{R} M^{*}$ where $M^{*}=\operatorname{hom}_{R}(M, R)$.

When $R$ is a prime maximal right order, we show that the endomorphism ring of any finite dimensional, reflexive module is a maximal order. We then show by example that $R$ being a maximal order is not a property preserved by $k$. However, we show that $k=$ End $M_{R}$ is a maximal order whenever $M_{R}$ is a maximal uniform right ideal of $R$, thereby sharpening Faith's representation theorem for maximal two-sided orders. In the final section, we show by example that even if $\boldsymbol{R}=$ End $_{k} V$ is a simple pli (pri)-domain, $k$ can have any prescribed right global dimension $\geqslant 1$, can be right but not left Noetherian or neither right nor left Noetherian.

In his survey, Jacobson [43] defined the concept of equivalence of two right orders $R_{1}$ and $R_{2}$ in a semisimple ring $Q$, namely $x_{1} R_{1} x_{2} \subseteq R_{2}$ and $y_{1} R_{2} y_{2}$ $\subseteq R_{1}$ for some choice of units $x_{1}, x_{2}, y_{1}, y_{2}$ of $Q$. In this paper we study when certain orders $R_{1}$ are maximal in the sense that $R_{1}=R_{2}$ whenever $R_{2}$ is an order equivalent to, and containing $R_{1}$.

If $M$ is any torsionless right $R$-module which is finite dimensional (in the sense of Goldie) then a theorem of Zelmanowitz [67] states that $k=$ End $M_{R}$ is an order in a semisimple ring in case $R$ is. We study this situation for a maximal two-sided order and show that $m=\operatorname{End}_{R} M^{*}$ is a maximal order whenever $M_{R}$ is finite dimensional, torsionless and faithful. As an immediate consequence we obtain the equivalence of the following conditions when $M_{R}$ is a generator:

1. $M$ is $R$-reflexive.

2. $k=$ End $M_{R}$ is a maximal order.

3. $k=\operatorname{End}_{R} M^{*}$ where $M^{*}=\operatorname{hom}_{R}(M, R)$.

Presented to the Society, January 15, 1974; received by the editors February 10, 1975. AMS (MOS) subject classifications (1970). Primary 16A18, 16A50, 16A64; Secondary 16A02, 16A04, 16A08, 16A46.

Key words and phrases. Order, equivalent order, maximal order, Goldie ring, Ore domain, principal left ideal ring, simple ring, torsionless module, reflexive module, projective module, generator, uniform module. 
When $R$ is a prime maximal right order and $M_{R}$ is reflexive, we show that $k=$ End $M_{R}$ is a maximal right order. We then show by example that $R$ being a maximal order is not a property preserved by $k$ even if $M$ is a uniform right ideal of $R$. However, we show that $k=$ End $M_{R}$ is a maximal order whenever $M_{R}$ is a maximal uniform right ideal of $R$. In this case, $k$ is in fact a right Ore domain. Consequently, we can sharpen Faith's representation theorem [72] for simple (maximal) orders $R$ by observing that $R=$ End ${ }_{k} V$ where ${ }_{k} V$ is a finitely generated, projective (finite dimensional, reflexive) $k$-module and $k$, a maximal order in a skew field.

In the final section we show by example that even if $A=$ End ${ }_{k} V$ is a simple pri (pli)-domain, then $k$ can have any prescribed right global dimension $\geqslant 1$, can be right but not left Noetherian or neither right nor left Noetherian. In the process we show that the existence of a certain type of simple ring implies a very pathological representation of $A$ and a partial answer to a question raised in Camillo-Cozzens [73].

I. Preliminaries. Throughout this paper, all rings are associative with identity, all modules and bimodules are unital. We shall always write homomorphisms on the opposite side of the scalars. If $A$ is any ring, $M \in \bmod -A$ or $M_{A}$ will signify that $M$ is a right $A$-module.

Since we shall repeatedly use them, we now summarize several more or less well-known facts. For details, see Faith [72]. Let $B$ be any ring, $U \in B$-mod and $A=$ End $_{B} U$. There is a canonical bimodule homomorphism

$$
\phi: \operatorname{hom}_{B}(U, B) \rightarrow \operatorname{hom}_{A}(U, A)
$$

defined by

$$
[v](\phi(f)(u))=(v) f \cdot u \quad \forall u, v \in U, f \in \operatorname{hom}_{B}(U, B) .
$$

By symmetry, if $B=$ End $U_{A}$, the map

$$
\psi: \operatorname{hom}_{A}(U, A) \rightarrow \operatorname{hom}_{B}(U, B)
$$

defined by

$$
((u) \psi(f))[v]=u \cdot f(v) \quad \forall u, v \in U, f \in \operatorname{hom}_{A}(U, A)
$$

is also a bimodule homomorphism. Moreover, in this case, $\phi$ is an isomorphism with inverse $\psi$.

Also recall that if $U_{A}$ is a generator and $B=$ End $U_{A}$, then $A=$ End ${ }_{B} U$ ( $U_{A}$ is balanced) and ${ }_{B} U$ is finitely generated projective. Moreover, ${ }_{A} U^{*}=$ $\operatorname{hom}_{A}(U, A) \approx \operatorname{hom}_{B}(U, B)$ is also a generator and $A \approx$ End $U_{B}^{*}$. If $T$ denotes the trace ideal of ${ }_{B} U$, then $T U=U$ and $T^{2}=T$. 
Recall that a module $U_{A}$ is torsionless if $U$ embeds canonically in its $A$-bidual $U^{* *}$ (equivalently, $U_{A}$ embeds in the direct product of $I$ copies of $A$ for some set $I$, and reflexive if $U_{A} \stackrel{\text { canonically }}{\approx} U_{A}^{* *}$.

Since the following result will be used repeatedly in the sequel and is perhaps, less familiar than the preceding, we shall include its proof.

1.1 Proposition. Let $V \in B_{0}-\bmod$ be faithful and torsionless, $B=$ Biend $B_{0} V$ (i.e., if $A=$ End $B_{0} V$, Biend $B_{0} V=$ End $\left.V_{A}\right), U^{0}=\operatorname{hom}_{B_{0}}\left(V, B_{0}\right)$ and $U=\operatorname{hom}_{B}(V, B)$. Then

a. the canonical inclusion $B_{0} \hookrightarrow B$ induces an inclusion $U^{0} \hookrightarrow U$.

b. $B T_{0} \subset B_{0}$ where $T_{0}=$ trace $_{B_{0}} V$. Hence, $B_{B_{0}}$ is an essential extension of $B_{O B_{0}}$.

c. $T_{0_{B_{0}}}$ is essential and ${ }_{B_{0}} T_{0}$ is essential whenever $B_{0}$ is semiprime.

Consequently, $U_{B_{0}}^{0}$ is necessarily faithful, implying an inclusion $B_{0} \hookrightarrow$ Biend $U_{B_{0}}^{0}$.

d. If ${ }_{B_{0}} V$ is finitely generated projective, $U^{0} T_{0}=U^{0}$.

Proof. a. Let $():, \operatorname{hom}_{B_{0}}\left(V, B_{0}\right) \rightarrow \operatorname{hom}_{A}(V, A)$ denote the canonical $\left(A, B_{0}\right)$-homomorphism $(\phi)$ defined above. For $b \in B, v, v^{\prime} \in V$ and $f \in U^{0}$, we have

$$
\begin{aligned}
(b v) f \cdot v^{\prime} & =(b v)\left(f, v^{\prime}\right)=b\left((v)\left(f, v^{\prime}\right)\right) \\
& =b\left((v) f \cdot v^{\prime}\right)=(b(v) f) \cdot v^{\prime}
\end{aligned}
$$

since $V$ is a $(B, A)$-bimodule. Since ${ }_{B} V$ is clearly faithful, $(b v) f=b(v) f$ $\forall b \in B, v \in V$ and $f \in U^{0}$. Thus, $U^{0} \subseteq U$ as asserted.

b. Clear in view of a.

c. Denote by $[v, f]=(v) f \forall v \in V, f \in U^{0}$ and by $\left[V, U^{0}\right]=\{[v, f] l v$ $\left.\in V, f \in U^{0}\right\}$. Clearly, $\left[V, U^{0}\right]=T_{0}$ and if $b T_{0}=0, b \in B_{0}, b\left[V, U^{0}\right]=$ $\left[b V, U^{0}\right]=0$. Since ${ }_{B_{0}} V$ is torsionless, $b V=0$ implying that $b=0$ since $B_{0} V$ is faithful. Therefore, $T_{0_{B_{0}}}$ is essential. If $T_{0} b=0,\left(b T_{0}\right)^{2}=0 \Rightarrow b T_{0}=$ $0 \Rightarrow b=0$ by semiprimeness of $B_{0}$, which clearly implies that ${ }_{B_{0}} T_{0}$ is essential in $B_{0} B_{0}$.

The last assertion follows readily from the equalities

$$
T_{0} b=\left[V, U^{0}\right] b=\left[V, U^{0} b\right] \quad \forall b \in B_{0} .
$$

d. Referring to the map $($, ) defined in a and setting

$$
\left(U^{0}, V\right)=\left\{(f, v) \mid f \in U^{0}, v \in V\right\}
$$

we have

$$
U^{0} \cdot\left[V, U^{0}\right]=\left(U^{0}, V\right) \cdot U^{0}=A U^{0}=U^{0}
$$


Here, we are using the well-known fact that $\left(U^{0}, V\right)=A$ iff ${ }_{B_{0}} V$ is finitely generated projective (Dual basis lemma).

As mentioned earlier, we shall be examining the representation of a simple Goldie ring (more generally, a maximal order) $A$ as the endomorphism ring of a finitely generated projective module ${ }_{B} V$ where $V_{A}$ is usually a uniform right ideal of $A$ or a finite dimensional (Goldie dimension) torsionless generator. The principal tool in our investigation will be the Faith Correspondence Theorem, some consequences of which we now state.

1.2 Proposition (FAITH). Let $V$ be finitely generated faithful and projective over a ring $B$, let $A=$ End $_{B} V$ and let $T=\operatorname{trace}_{B} V$. Then

(1) $A$ has a finite ideal lattice if and only if the set of ideals TIT of $B$ is finite. In particular, $A$ is a simple ring if and only if $T K=0$ for every ideal $K \neq T$, contained in $T$.

(2) If $B$ is semiprime, then $A$ is simple if and only if $T$ is the least ideal of $B$. In this case a right ideal $I$ of $B$ satisfies $I=I T$ if and only if $I$ is an idempotent right ideal contained in $T$.

(3) $\operatorname{dim} V_{A}=\operatorname{dim} B_{B}$ and $\operatorname{dim} A_{A}=\operatorname{dim}\left(\operatorname{hom}{ }_{B}(V, B)\right)_{B}\left(\operatorname{dim} V_{A}\right.$ is always the Goldie dimension of $V_{A}$ ).

II. Maximal orders. Throughout this section, all rings will be at least • right orders in semisimple rings, i.e., semiprime right Goldie rings. The term order will be reserved for a semiprime two-sided Goldie ring.

2.1 Definition. Let $R_{1}$ and $R_{2}$ be right orders in a ring $Q$. Then

(i) they are equivalent, $R_{1} \stackrel{Q}{\sim} R_{2}$, if there exist regular elements $a_{1}, b_{1}, a_{2}$, $b_{2}$ of $Q$ such that $a_{1} R_{1} b_{1} \subseteq R_{2}$ and $a_{2} R_{2} b_{2} \subseteq R_{1}$;

(ii) they are right equivalent, $R_{1} \stackrel{r}{\sim} R_{2}$ if there exist regular elements $a_{1}$, $a_{2}$ of $Q$ such that $a_{1} R_{1} \subseteq R_{2}$ and $a_{2} R_{2} \subseteq R_{1}$;

(iii) they are left equivalent, $R_{1} \sim R_{2}$ if there exist regular elements $b_{1}, b_{2}$ of $Q$ such that $R_{1} b_{1} \subseteq R_{2}$ and $R_{2} b_{2} \subseteq R_{1}$.

A right order $R$ is maximal (left equivalent, right equivalent) provided that $R \subseteq R^{\prime} \subseteq Q$ and $R \sim R^{\prime}\left(R \stackrel{l}{\sim} R^{\prime}, R \stackrel{r}{\sim} R^{\prime}\right)$ imply that $R=R^{\prime}$.

2.2 Proposition. a. Let $R, S$ be right orders with $R \subseteq S, R \sim S$. There exist right orders $T, T$ 'such that

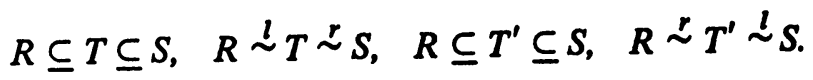

b. $R$ is maximal iff $R$ is a maximal left and right equivalent order.

Proof. Consult Faith [73].

REMARK. Any simple ring is a maximal order. 
2.3 Proposition. Let $R$ be a maximal semiprime right Goldie ring, $M_{R}$ a finite dimensional torsionless generator and $k=$ End $M_{R}$. If $k^{\prime} \supset k$ and $k^{\prime} \mathcal{L}$ $k$, then $R=$ End $k^{\prime} k^{\prime} \otimes_{k^{\prime}} M$.

Proof. Set $M^{\prime}=k^{\prime} \otimes_{k^{M}} M$ and $R^{\prime}=\operatorname{End}_{k^{\prime}} M^{\prime}$. Since $k^{\prime} M^{\prime}$ is finitely generated and projective, $M_{R^{\prime}}^{\prime}$ is a generator and hence, balanced i.e., if $k^{\prime \prime}=$ End $M_{R}^{\prime}, R^{\prime}=$ End $_{k^{\prime \prime}} M^{\prime}$. Moreover, if $k^{M}=k^{n} e, k^{\prime} M^{\prime}=k^{\prime n} e$ and $R^{\prime}=e k_{n}^{\prime} e$ implying that $R^{\prime}$ is an order in the quotient ring of $R$. Thus, End $M_{R^{\prime}}^{\prime}=$ End $M_{R}^{\prime}$. Since $\alpha k^{\prime} \subseteq k$, where $\alpha$ is a regular element of $k, \alpha$ induces a monomorphism $M_{R}^{\prime} \rightarrow$ $M_{R}$ implying that $M_{R}^{\prime}$ is finite dimensional, torsionless and hence, $T=\operatorname{trace} M_{R}^{\prime}$ $\neq 0$. By earlier remarks, since $M_{R}^{\prime}$ is clearly faithful, $T R^{\prime} \subseteq R$. However, by 1.1, $T_{R}$ is essential since $M_{R}^{\prime}$ is faithful and torsionless. Thus, $T$ contains a regular element of $R$ implying that $R^{\prime} \stackrel{\sim}{\sim}$ and hence $R^{\prime}=R$ by maximality of $R$.

2.4 Proposition. Let $R$ be a maximal order, $M_{R}$ a finite dimensional, torsionless, faithful $R$-module, $k=$ End $M_{R}, M^{*}=\operatorname{hom}_{R}(M, R)$ and $M^{\#}=$ $\operatorname{hom}_{k}(M, k)$. Then

a. End $M_{k}^{\#}=$ End $M_{k}^{*}=R$.

b. $k^{\prime}=\operatorname{End}_{R} M^{\#}=\operatorname{End}_{R} M^{*}$ is an essential extension of $k$ and hence, $a$ right equivalent order in the quotient ring of $k$.

c. $\operatorname{dim}_{R} M^{*}=\operatorname{dim} M_{R}$.

d. $M^{*} \approx M^{* * *}$.

PRoof. Since $M_{R}$ is clearly balanced by maximality of $R, M^{\#}$ is canonically isomorphic to $M^{*}$ and hence, $\operatorname{End}_{R} M^{\#}$ can be identified with $\operatorname{End}_{R} M^{*}$.

a. Set $R^{\prime}=$ End $M_{k}^{*} \cdot M R^{\prime} M^{*}=M M^{*} \subseteq k \Rightarrow M R^{\prime} M^{*} M=M R^{\prime} T \subseteq M \Rightarrow$ $R^{\prime} T \subseteq R \Rightarrow R^{\prime} \stackrel{l}{\sim} R$ by 1 .1c. Since $R^{\prime} \supseteq R, R^{\prime}=R$ by maximality of $R$.

b. Since $k^{\prime}=$ Biend $M_{k}^{*}, T k^{\prime} \subseteq k$ where $T=$ trace $M_{k}^{*}$. Hence, $k^{\prime}$ is an essential extension of $k$ and, since $T$ is an essential right ideal of $k, T$ contains a regular element of $k$ and thus, $k^{\prime}{ } k$.

c. Set $\bar{M}=M \oplus R, \bar{k}=$ End $\bar{M}_{R}$ and $\bar{k}^{\prime}=\operatorname{End}_{R} \bar{M}^{*}$. Clearly, $\bar{M}_{R}$ is a generator, hence, $\operatorname{dim} \bar{M}_{R}=\operatorname{dim} \bar{k}_{\bar{k}}$ and $\operatorname{dim}_{R} \bar{M}^{*}=\operatorname{dim}_{\bar{k}^{\prime}} \bar{k}^{\prime}$ by 1.2.3. Since both $\bar{k}$ and $\bar{k}^{\prime}$ are orders and $\bar{k}_{\bar{k}}^{\prime}$ is an essential extension of $\bar{k}_{\bar{k}}$ by b, $\operatorname{dim} \bar{k}_{\bar{k}}=$ $\operatorname{dim}_{\bar{k}} \bar{k}=\operatorname{dim}_{\bar{k}} \bar{k}^{\prime}=\operatorname{dim}_{\bar{k}^{\prime}} \bar{k}^{\prime}$. Thus, $\operatorname{dim}_{R} \bar{M}^{*}=\operatorname{dim} \bar{M}_{R}$ which clearly implies that $\operatorname{dim}_{R} M^{*}=\operatorname{dim} M_{R}$ as claimed.

d. By c, $\operatorname{dim}_{R} M^{*}=\operatorname{dim} M_{R}^{* *}=\operatorname{dim}_{R} M^{* * *}$. Since ${ }_{R} M^{*}$ is isomorphic to a direct summand of ${ }_{R} M^{* * *}$ (see Jans [64, p. 67]), we have ${ }_{R} M^{*} \approx{ }_{R} M^{* * *}$.

The next lemma allows us to assume in many instances that the module in question is a generator. The idea is due to Jategaonkar [71].

2.5 Lemma. Let $R$ be a semiprime right Goldie ring, $M$ a finite dimensinal, torsionless, faithful $R$-module and $k=$ End $M_{R}$. Then $k$ is a maximal 
(right equivalent, left equivalent) order whenever $\mathrm{End}(M \oplus R)_{R}$ is a maximal (right equivalent, left equivalent) order.

Proof. Viewing the elements of $M \oplus R$ as column vectors,

$$
\bar{k}=\text { End }(M \oplus R)_{R}=\left(\begin{array}{cc}
k & M \\
M^{*} & R
\end{array}\right)
$$

and its quotient ring $\bar{D}$ is

$$
\bar{D}=\operatorname{End}\left((M \oplus R) \otimes_{R} Q\right)_{Q}=\left(\begin{array}{cc}
\operatorname{End}\left(M \otimes_{R} Q\right)_{Q} & M \otimes_{R} Q \\
\operatorname{hom}_{Q}\left(M \otimes_{R} Q, Q\right) & Q
\end{array}\right)
$$

where $Q=$ the quotient ring of $R$. Furthermore, $D=\operatorname{End}\left(M \otimes_{R} Q\right)_{Q}=$ the quotient ring of $k$ (see Zelmanowitz [67]).

Suppose $\bar{k}$ is maximal and $k^{\prime} \supseteq k$ with $k^{\prime} \sim k$, say, $\alpha k^{\prime} \beta \subseteq k$ with $\alpha$ and $\beta$ regular elements of $k$. Set

$$
S=\left(\begin{array}{ll}
k^{\prime} & k^{\prime} M \\
M^{*} k^{\prime} & M^{*} k^{\prime} M
\end{array}\right)+\bar{k}
$$

$S$ is clearly an order in $\bar{D}$ containing $\bar{k}$. Since

$$
\bar{k}\left(\begin{array}{ll}
\alpha & 0 \\
0 & 0
\end{array}\right) \bar{k} S \bar{k}\left(\begin{array}{ll}
\beta & 0 \\
0 & 0
\end{array}\right) \bar{k} \subseteq \bar{k}
$$

(routine computation), $S \sim \bar{k}$ if the ideals $\bar{k}\left(\begin{array}{ll}\alpha & 0 \\ 0 & 0\end{array}\right) \bar{k}$ and $\bar{k}\left(\begin{array}{ll}\beta & 0 \\ 0 & 0\end{array}\right) \bar{k}$ contain regular elements of $\bar{k}$. To demonstrate this, we shall consider only the first ideal. Since $\bar{k}\left(\begin{array}{ll}\alpha & 0 \\ 0 & 0\end{array}\right) \bar{k}$ contains all matrices of the form

$$
\left(\begin{array}{cc}
k \alpha k & 0 \\
0 & {\left[M^{*}, \alpha M\right]}
\end{array}\right)
$$

such a regular element exists provided the ideal $I=\left[M^{*}, \alpha M\right]$ is essential as a right ideal of $R$; equivalently, $I r=0 \Rightarrow r=0$ (see the proof of 1.1c). If $I r=0, \alpha M r=0$ since $M$ is torsionless. Thus, $M r=0$ ( $\alpha$ is regular) $\Rightarrow r=0$ since $M$ is faithful. Consequently, $I$ is essential as asserted.

The proofs of the parenthetical statements are special cases of the above.

When $R$ is prime, Hart-Robson [70] have shown that $e R_{n} e$ is maximal for all idempotents $e \in R_{n}$ and $n \geqslant 1$. The argument used in the proof of 2.5 is essentially theirs.

2.6 LeMmA. Let $R$ be a semiprime right Goldie ring, $M_{R}$ a finite dimensional, torsionless generator, $k=\operatorname{End} M_{R}$ and $m=\operatorname{End}_{R} M^{*}$. If $k^{\prime}$ is any ring containing $k$ with $R=\operatorname{End}_{k^{\prime}} k^{\prime} \otimes M$ and hom $_{R}\left(k^{\prime} / k \otimes_{k^{\prime}} M, R\right)=0$, then $k^{\prime} \subseteq \underline{m}$. 
Proof. Set $M^{\prime}=k^{\prime} \otimes_{k} M$. Since $M_{R}$ is a generator and $R=$ End $_{k^{\prime}} M^{\prime}$, the canonical maps $M^{\#}=\operatorname{hom}_{k}(M, k) \rightarrow \operatorname{hom}_{R}(M, R)=M^{*}$ and $\operatorname{hom}_{k^{\prime}}\left(M^{\prime}, k^{\prime}\right) \rightarrow$ $\operatorname{hom}_{R}\left(M^{\prime}, R\right)$ are an isomorphism and a monomorphism, respectively. Since $\operatorname{hom}_{R}\left(M^{\prime} / M, R\right)=0$, the canonical map $\operatorname{hom}_{R}\left(M^{\prime}, R\right) \rightarrow \operatorname{hom}_{R}(M, R)$ is an embedding. One readily checks that the composition of maps

$$
\operatorname{hom}_{k^{\prime}}\left(M^{\prime}, k^{\prime}\right) \rightarrow \operatorname{hom}_{R}\left(M^{\prime}, R\right) \hookrightarrow \operatorname{hom}_{R}(M, R) \rightarrow M^{\#}
$$

is the map defined by

$$
\left.\left.f \rightarrow f\right|_{M} \quad \text { (restriction to } M\right) \quad \forall f \in \text { hom }_{k^{\prime}}\left(M^{\prime}, k^{\prime}\right) \text {. }
$$

Thus, any $k$-linear map $f: M \rightarrow k^{\prime}$ is actually a map into $k$ since $f$ induces a $k^{\prime}$-linear $\bar{f}: M^{\prime} \rightarrow k^{\prime}$ such that $\left.\bar{f}\right|_{M}=f$.

Next, if $T=\operatorname{trace}_{k} M, T k^{\prime} \subseteq k$. For, if $(u) f \in T, \alpha^{\prime} \in k^{\prime}, f \alpha^{\prime} \in \operatorname{hom}_{k}\left(M, k^{\prime}\right)$ $=\operatorname{hom}_{k}(M, k)$ by the above remarks. Hence, $(u) f \alpha^{\prime} \in k$. Finally, $M^{\#} T k^{\prime}=$ $M^{\#} k^{\prime} \subseteq M^{\#}$ which implies that $k^{\prime} \subseteq m$.

2.7 THEOREM. Let $R$ be a maximal order and $M$ a finite dimensional, torsionless, faithful $R$-module. Then $m=\operatorname{End}_{R} M^{*}$ is amaximal order and is the maximal right equivalent order containing $k$.

PROoF. It suffices to show that whenever $k^{\prime} \supset k=$ End $M_{R}$ and $k^{\prime} \stackrel{\boldsymbol{r}}{\sim} k, k^{\prime} \subseteq$ $m$ and if $k^{\prime} \supseteq m$ with $k^{\prime} \downarrow_{m}, k^{\prime} \subseteq m$. For if this condition is satisfied and $k^{\prime} \supseteq m$ with $k^{\prime} \sim m$, then $k^{\prime} \sim k$ and by 2.2 there exists an order $s$ with $k^{\prime} \supseteq s \supseteq k, k^{\prime} \downarrow_{s}^{-}$and $s \mathcal{L}_{k}$. By assumption, $s \subseteq m$ and since $k^{\prime} \downarrow_{s, k^{\prime}} \downarrow_{m}$. Thus, $k^{\prime} \subseteq m$, and $m$ is maximal as claimed.

To show that the above condition is fulfilled, we can clearly assume that $M$ is a generator by 2.5 . If $k^{\prime} \supset k$ with $k^{\prime} \mathcal{L}_{k}$, set $M^{\prime}=k^{\prime} \otimes_{k} M$. By $2.3, R=\operatorname{End}_{k^{\prime}} M^{\prime}$ and since $M^{\prime}$ is an essential extension of $M, \operatorname{hom}_{R}\left(M^{\prime} / M, R\right)=0$. Hence by 2.6 , $k^{\prime} \subseteq m$.

In order to complete the proof, suppose that $k^{\prime} \supseteq m$ with $k^{\prime} \downarrow_{m}$. Then by an obvious modification of 2.3,R $=$ End $\left(M^{\#} \otimes_{m} k^{\prime}\right)_{k^{\prime}}$. By modifying the above argument, we obtain hom ${ }_{m}\left(M^{\#}, k^{\prime}\right)=\operatorname{hom}_{m}\left(M^{\#}, m\right)$ and hence, $k^{\prime} T^{\#} \subseteq m$ where $T^{\#}=\operatorname{trace} M_{m}^{\#}$. Since $m=$ End $M_{R}^{* *}$ (see the proof of $1 \Rightarrow 3$ in 2.8) and $T^{\#} M^{* *}$ $=M^{* *}, k^{\prime} \subseteq m$ as claimed.

2.8 THEOREM. Let $R$ be a maximal order and $M$ a finite dimensional, torsionless, faithful $R$-module. Then $1 \Rightarrow 2 \Longleftrightarrow 3$, where

1. $M_{R}$ is $R$-reflexive,

2. $k=$ End $M_{R}$ is a maximal order,

3. $k=$ End $_{R} M^{*}$ where $M^{*}=\operatorname{hom}_{R}(M, R)$.

Moreover, if $M$ is a generator, then $2 \Rightarrow 1$. 
Proof. We shall first assume that $M$ is a generator and prove the stated equivalences for this case. Then we shall discuss how the general case can be reduced to the case of a generator.

$$
\begin{aligned}
& (1 \Rightarrow 3): \text { Since } M=M^{* *}, \\
& k=\text { End } M_{R}=\operatorname{hom}_{R}\left(M, M^{* *}\right)=\operatorname{hom}_{R}\left(M^{*}, \operatorname{hom}_{R}(M, R)\right)=\operatorname{End}_{R} M^{*} .
\end{aligned}
$$

The third equality follows from one of the standard functional isomorphisms (e.g., see Cartan-Eilenberg [56, Exercise 4, p. 32]).

$(3 \Longleftrightarrow 2)$ : Immediate by 2.4 and 2.7 .

$(2 \Rightarrow 1):$ By $2.4, k^{\prime}=$ End $_{R} M^{\#}=k$. Since $M^{\#} \approx M^{*}$ and hom $k^{\prime}\left(M^{\#}, k^{\prime}\right) \approx$ $\operatorname{hom}_{R}\left(M^{*}, R\right)=M^{* *}, M^{\# \#} \approx M^{* *}$ as $(k, R)$-bimodules. By finite projectivity of ${ }_{k} M, M^{\# \#} \approx M$ as $(k, R)$-bimodules. Hence, $M$ is $R$-reflexive as asserted.

If $M$ is no longer assumed to be a generator, note that $M$ is reflexive iff $\bar{M}=$ $M \oplus R$ is reflexive, and End $\bar{M}_{R}$ is maximal only if End $M_{R}$ is maximal. Moreover, $\bar{M}$ is always a generator for arbitrary $M$. Thus, $1 \Rightarrow 2$, and $2 \Rightarrow 3$ follows immediately from 2.4b. If $k=$ End $_{R} M^{*}$, then $k$ is maximal by 2.7 . Consequently, $3 \Rightarrow 2$.

REMARK. To see that $2 \Rightarrow 1$ does not hold in general, let $R$ be any maximal order having a faithful ideal $I$ which is not reflexive as a right ideal and set $k=$ End $I_{R}$; e.g., any $n$-dimensional regular local ring with $n \geqslant 2$. These are maximal orders by Auslander-Goldman [60] and must have nonreflexive right ideals by Matlis [68]. Since $k \supseteq R$ and $k \sim R, k=R$. Thus, $I_{R}$ is not reflexive but $k$ is maximal.

In order to generalize the above to maximal right orders, care must be exercised to insure that all of the endomorphism rings in question are right orders in the appropriate semisimple ring. For example, if $M_{R}$ is a finite dimensional,torsionless generator, there is no guarantee that $k^{\prime}=$ End $_{R} M^{*}$ is a right order in the quotient ring of $k$. However, when $R$ is prime and $M_{R}$ reflexive, we can conclude that $k$ is maximal. Specifically,

2.9 Proposition. Let $R$ be a prime maximal right order, $M_{R}$ a finite dimensional reflexive $R$-module and $k=\operatorname{End} M_{R}$. Then $k$ is a maximal right order.

Proof. Since 2.5 is valid for right orders, it suffices to assume that $M_{R}$ is a generator. If $k^{\prime} \iota_{k}$ or $k^{\prime} L_{k}$, since hom ${ }_{R}\left(k^{\prime} / k \otimes_{k} M, R\right)=0$ in either case, $k^{\prime} \subseteq$ $m=$ End $_{R} M^{*}$ provided $R=$ End $_{k^{\prime}} k^{\prime} \otimes_{k} M$. This, of course, implies that $k$ is maximal since $m=k$. (Proof of $1 \Rightarrow 3$ in 2.8.) Thus, it suffices to show that if $k^{\prime} \stackrel{l}{L}_{k, R}=\mathrm{End}_{k^{\prime}} k^{\prime} \otimes M\left(2.3\right.$ covers the case where $\left.k^{\prime} \iota_{k}\right)$.

To that end note that for some $n>0, R^{\prime}=$ End $_{k}, k^{\prime} \otimes M \approx e k_{n}^{\prime} e$ and $R \approx$ $e k_{n} e$. Since $k^{\prime} \alpha \subseteq k, e k_{n}^{\prime} e k_{n} e k_{n} \bar{\alpha} e k_{n} e \subseteq e k_{n} e$ where $\bar{\alpha}=\alpha I_{n}, I_{n}$ the $n \times n$ identity matrix. Since $e k_{n} e$ is prime, the ideal $e k_{n} e k_{n} \bar{\alpha} e k_{n} e$ is essential and hence, contains 
a regular element of $e k_{n} e$. Thus, $e k_{n}^{\prime} e \stackrel{r}{\sim} e k_{n} e \Rightarrow e k_{n}^{\prime} e=e k_{n} e$ by maximality of $R\left(e k_{n} e\right)$.

The question of whether one-sided versions of 2.7 and 2.8 hold for general semiprime $R$ remains open. If $k^{\prime} \stackrel{L}{\sim} k$ always implies $e k_{n}^{\prime} e \stackrel{\perp}{\sim} e k_{n} e$, then, of course, the above argument shows that finite dimensional, faithful, reflexive modules have maximal endomorphism rings.

As an interesting aside, note that the proof of 2.9 shows that $m=k_{T}$, the (left) quotient ring of $k$ with respect to the smallest filter of left ideals which contain $T=\operatorname{trace}_{k} M$.

III. The case of global dimension at most 2. In order to show that reflexive generators, specifically in the case of maximal orders, are bonafide generalizations of finitely generated projective generators, we shall show (3.2) that if each finitely generated reflexive module is projective, then the global dimension of the ring cannot exceed 2 (the converse is well known). Then, if $U_{R}$ is any finitely generated reflexive, nonprojective (necessarily gld $R>2$ ) $U_{R} \oplus R_{R}$ is a nonprojective, reflexive generator. For simplicity, we shall assume that $R$ is a semiprime two-sided Noetherian ring. To prove the above assertion, we need the following characterization of two-sided Noetherian rings of global dimension $\leqslant 2$.

3.1 Proposition (BASS [60]). If $R$ is left and right Noetherian, the following are equivalent:

(1) gld $R \leqslant 2$.

(2) The dual of any finitely generated right $R$-module is projective.

Let $M_{R}$ be any finitely generated $R$-module and $t M$ its torsion submodule. We can assume that $M_{R}$ is a generator.by the remarks preceding the conclusion of the proof of 2.8 . Clearly, $M^{*}=(M / t M)^{*}$ since $(t M)^{*}=0$. Thus, we can further assume that $M$ is a torsion free and hence, a torsionless, finitely generated $R$-module. Since $M_{R}$ is clearly finite dimensional, ${ }_{R} M^{*} \approx{ }_{R} M^{* * *}$ and assuming reflexive modules are projective, $M^{*}$ is projective. Consequently, by 3.1 , gld $R$ $\leqslant 2$. Thus, we have shown that

3.2 Proposition. The following conditions are equivalent for any semiprime two-sided Noetherian ring $R$ :

1. gld $R \leqslant 2$.

2. Each finitely generated reflexive module is projective.

A canonical and important example of a reflexive module is given by

3.3 Proposition. Any maximal uniform right ideal of $R$ (more generally, any right annulet) is reflexive. 
Proof. By Bass [60], $U=X^{*}$ for some finitely generated left $R$-module $X$. Since we can assume that $X_{R}^{*}$ is a generator, $\operatorname{dim} X_{R}^{*}=\operatorname{dim} X_{R}^{* * *}$ and consequently, $U=X^{*}=X^{* * *}=U^{* *}$.

By parroting an argument used in Proposition 5.5 of Faith [72], we readily obtain the necessity of the following characterization of simple Noetherian domains having global dimension less than or equal to 2 .

3.4 Theorem. a. Let $R$ be a simple, two-sided Noetherian domain. Then gld $R \leqslant 2$ iff $\forall n \geqslant 1$, whenever $B$ is a maximal order and $B \sim R_{n}, B$ is simple. b. If $R$ is simple Noetherian with gld $R \leqslant 2, M_{R}$ finite dimensional and torsionless, then $k^{\prime}=\operatorname{End}_{R} M^{*}$ is a simple Noetherian ring Morita equivalent to $R$.

Proof. b. Since $M_{R}$ is finite dimensional and torsionless, $M_{R} \hookrightarrow R^{(n)}$ for some $n \geqslant 1$, implying that $T=\operatorname{trace} M_{R} \neq 0$. By simplicity of $R, T=R$ and hence, $M_{R}$ is a generator. Therefore, by $3.1_{R} M^{*}$ is a progenerator and hence, $k^{\prime}$ is Morita equivalent to $R$.

a. (Necessity): We shall present only the $n=1$ case. The general case is identical. Suppose $x B y \subseteq R$. Set $a=x y$ and $B^{\prime}=x B x^{-1}$. Then $B^{\prime} a \subseteq R$ and hence, $I=B^{\prime} a R$ is a right ideal of $R$ satisfying $B^{\prime} \subseteq$ End $I_{R}$. Since End $I_{R} \sim R$, $B^{\prime}=$ End $I_{R}$ by maximality of $B^{\prime}$ ( $B^{\prime}$ maximal iff $B$ is). Moreover, $B^{\prime}=$ End ${ }_{R} I^{*}$ by 2.4 . Since gld $R \leqslant 2,{ }_{R} I^{*}$ is a progenerator implying that $B^{\prime}$ and hence, $B$ is simple.

(Sufficiency): By 3.2 it suffices to show that each finitely generated, reflexive $R$-module is projective. To that end suppose $I_{R}$ is finitely generated reflexive and $\operatorname{dim} I_{R}=n$. Then clearly $R^{(n)} \hookrightarrow I_{R}$ and since $\operatorname{dim}{ }_{R} I^{*}=n, I_{R} \hookrightarrow R^{(n)}$. This readily implies that $B=$ End $I_{R} \sim R_{n}=$ End $R_{R}^{(n)}$. B is a maximal order since $I_{R}$ is a reflexive generator and hence, simple by hypothesis. Finally, by simplicity of $B, I_{R}$ is projective, completing the proof of 3.4.

As a final remark regarding uniform right ideals we have

3.5 PROPOSITION. If $R$ is a simple, two-sided, Noetherian ring satisfying gld $R \leqslant 2$ and each uniform right ideal is reflexive, then $R$ is hereditary.

Proof. We can assume $R$ is a domain by 3.4. Since each right ideal is now reflexive, each right ideal is projective by 3.1 .

Thus, even in such well-behaved rings, one cannot expect to find too many reflexive uniform right ideals.

IV. The nonreflexive case. The object of this section is to provide further insight into the structure of the endomorphism ring of a finite dimensional torsionless $R$-module $M$, in particular, a basic right ideal of $R$, and to sharpen Faith's representation theorem mentioned in the introduction. In 4.1, we shall assume 
that $R$ is simple. While $4.1 \mathrm{~b}$ is 2.7 in a less general setting, its proof is an interesting application of the Correspondence Theorem.

4.1 Proposition. Let $R$ be a simple order, $M_{R}$ a finite dimensional, torsionless $R$-module and $k=$ End $M_{R}$. Then

a. $k$ is a maximal left equivalent order;

b. $m=\operatorname{End}_{R} M^{*}$ is the maximal equivalent order containing $k$.

PROof. a. $M_{R}$ is necessarily a generator for $\bmod -R$. Let $T=\operatorname{trace}_{k} M$. If $k^{\prime} \supseteq k$ with $k^{\prime} \downarrow_{k}$, say $k^{\prime} \alpha \subseteq k$, then $k^{\prime} \alpha T=T=k^{\prime} T$ since $T$ is the least ideal of $k$. Thus, $k^{\prime} T M=k^{\prime} M \subseteq M$ and hence $k^{\prime} \subseteq k$.

b. If $k^{\prime} \supset k$ with $\alpha k^{\prime} \subseteq k, T \alpha k^{\prime}=T=T k^{\prime} \Rightarrow k^{\prime} \subseteq m$ since $M^{*} T=M^{*}$.

Thus, any equivalent order containing $k$ is contained in $m$ implying that $m$ is the maximal such order.

4.2 Proposition. (1)(FAith [64]) Any maximal order in $Q=D_{n}, D$ a field, is isomorphic to the biendomorphism ring of any right ideal $U$, say $R \approx \operatorname{End}_{k} U$ where $k=$ End $U_{R}$.

(2) If $U_{R}$ is a maximal uniform right ideal, then $k=$ End $U_{R}$ is a maximal order in $D$.

Proof. (1) Let $U$ be any right ideal of $R$. By $1.1, T=$ trace $U_{R}$ satisfies $T \bar{R} \subseteq R$ where $\bar{R}=$ Biend $U_{R}$. Since $T$ contains a regular element of $R, \bar{R}$ is a right order of $Q$ which is $\stackrel{r}{\sim}$ to $R$. Thus, $\bar{R}=R$ by maximality of $R$.

(2) Clear.

V. Examples. In this section, we shall show that if $A=\operatorname{End}_{B} V$ where ${ }_{B} V$ is finitely generated projective over a right Ore domain $B, A$ simple, the domain $B$ can be quite "bad" when $A$ is quite "good".

Recall the definition of the ring of linear differential operators $\mathfrak{D}=\mathfrak{D}_{R}$ over a ring $R$ with derivation $\delta$ :

$$
(\mathfrak{D},+)=(R[\delta],+)
$$

multiplication in $\mathbb{D}$ is induced by the relations

$$
\delta a=a \delta+\delta(a) \quad \forall a \in R .
$$

It is easy to show that whenever $R=k$ is a field of characteristic 0 and $\delta$ is outer, $D$ is a simple pli (pri)-domain. More generally, if $\operatorname{dim} k_{\operatorname{con} \delta}=\infty$ and $\delta$ is outer, then $D$ is simple.

In the following examples, we shall freely use results from Robson [72] and certain generalizations which can be found in Goodearl [73]. These references will be abbreviated to [72] and [73] respectively.

For any $n \geqslant 2$, there exists a right but not left Noetherian domain $B$ with 
1.gld $B=$ r.gld $B=n$, a finitely generated projective left $B$-module ${ }_{B} V$ such that $A=\operatorname{End}_{B} V$ is a simple pri (pli)-domain.

EXAMPLE 1. Let $k \supset k^{\prime}$ be fields with $1 . \operatorname{dim}_{k^{\prime}} k=\infty$ and r.dim $k_{k^{\prime}}$ finite (see Cohn [61] for such examples). Set $A=\mathfrak{D}_{k(x)}$ where $\delta$ is defined in the obvious fashion:

$$
\delta(x)=1, \quad \delta(a)=0 \quad \forall a \in k
$$

By Example 7.3 in [72], the subring $B=k+\delta A$ of $A$ is a hereditary Noetherian domain. By Theorem 4.3 of [73], $B^{\prime}=k^{\prime}+\delta A$ is a two-sided order in $A$ with r.gld $B^{\prime}=1$.gld $B^{\prime}=2$. Moreover, $B^{\prime}$ is right Noetherian and not left Noetherian. $\operatorname{End}_{B} \cdot V$.

Finally, set $V=\delta A$. Clearly, ${ }_{B^{\prime}} V$ is finitely generated projective and $A=$

By an obvious modification of this technique, we can choose $B^{\prime}$ such that $B^{\prime}$ is a two-sided order in $A, B^{\prime}$ is right but not left Noetherian and finally

$$
\text { r.gld } B^{\prime}=1 . \text { gld } B^{\prime}=n \text {. }
$$

Moreover, $B^{\prime}$ can be chosen to be neither right nor left Noetherian but still a twosided order, having any prescribed global dimension.

ExAMPle 2. Let $R$ be a pri-domain, $D$ its right quotient field and suppose that $D / R$ is semisimple in mod-R. Then there exists a right hereditary, right Noetherian domain $B$, which is, however, a two-sided order, a finitely generated projective ${ }_{B} V$ such that $A=\operatorname{End}_{B} V$ is a simple pri (pli)-domain.

Set $S=D_{D(x)}$ where $\delta$ is the standard formal derivative of $D(x), T=D+$ $\delta S$ and $k=R+\delta S$. As before, $T$ is a hereditary Noetherian domain. Also $k \sim S$ since $\delta S \subset k$. Thus, $k$ is a two-sided order in $F=$ right quotient field of $S$. We claim that $k$ is right hereditary. First, one readily verifies that the exact sequence of $k$-modules

$$
0 \rightarrow T / \delta S \rightarrow S / \delta S \stackrel{\pi}{\rightarrow} S / T \rightarrow 0
$$

is split exact (the obvious map is the desired right inverse to $\pi$ ) and hence, $\operatorname{pd}(S / T)_{k} \leqslant \operatorname{pd}(S / \delta S)_{k}$. Since $S_{k}$ is projective by Lemma 2.1 in [72], $\operatorname{pd}(S / \delta S)_{k}$ $\leqslant 1$ and hence, $\operatorname{pd}(S / T)_{k} \leqslant 1$, implying that $T_{k}$ is projective. Since $k$ is an order in $T$, it is easy to see that $T \otimes_{k} T=T$. As an immediate consequence, we have $\operatorname{pd} M_{k}=\operatorname{pd} M_{T}$ for all $M \in \bmod -T$ by a proof similar to that of Lemma 2.8 in [72]. Moreover, since $D / R$ is semisimple in mod-R and isomorphic to $T / k$, $T / k$ is semisimple in mod- $R$ and hence, in mod-k.

Finally, let $I$ be any right ideal of $k$. Then $I T$ is projective in mod-k and $I T / I$ is a direct summand of a coproduct of copies of $T / k$. Since $T_{k}$ is projective, 
pd $T / k<1$ implying that $\mathrm{pd} I T / I \leqslant 1$. Hence, $\mathrm{pd} I_{k}=0$. Thus, $k$ is right hereditary and since it is an order in a field, $k$ is right Noetherian.

Note that if we set $V=\delta S, S=\operatorname{End}_{k} V$. We also remark that such rings as $R$ exist. For example the rings discussed in Cozzens [70] work. The existence of an example principal on the right but not on the left remains an open problem. However, I feel very strongly that such examples do exist. Note that $R$ in Example 2 does not have to be left Ore! Finally, if such an $R$ exists (which is not left Noetherian), then $k$ affords us with an example of a two-sided order which is right hereditary but not left hereditary, thus answering a question raised in Camillo-Cozzens [73].

VI. Concluding remarks. By parroting the arguments used by Matlis [68], one trivially shows that if $R$ is a two-sided Noetherian ring with finite global dimension, then $R$ is hereditary if and only if each right ideal is $R$-reflexive. In the case of a maximal order, it would be interesting to know if a one-sided version of the above holds. More generally, what does the reflexivity of each uniform right ideal imply?

ACKNOWLEDGEMENT. The author is deeply indebted to Frank Sandomierski for discovering several "sticky points" in the original version of this paper and for many stimulating discussions about maximal orders.

\section{BIBLIOGRAPHY}

[60] M. Auslander and O. Goldman, Maximal orders, Trans. Amer. Math. Soc. 97 (1960), 1-24. MR 22 \#8034.

[60] H. Bass, Finitistic dimension and a homological generalization of semiprimary rings, Trans. Amer. Math. Soc. 95 (1960), 466-488. MR 28 \#1212.

[73] V. Camillo and J. H. Cozzens, $A$ theorem on noetherian hereditary rings, Pacific J. Math. 45 (1973), 35-41. MR 47 \#6745.

[56] H. Cartan and S. Eilenberg, Homological algebra, Princeton Univ. Press, Princeton, N. J., 1956. MR 17, 1040.

[61] P. M. Cohn, Quadratic extension of skew flelds, Proc. Londoh Math. Soc. (3) 11 (1961), 531-556. MR $25 \# 101$.

[70] J. H. Cozzens, Homological properties of the ring of differential polynomials, Bull. Amer. Math. Soc 76 (1970), 75-79. MR 41 \#3531.

[64] C. C. Faith, Noetherian simple rings, Bull. Amer. Math. Soc. 70 (1964), 730731. MR $29 \# 4773$.

[72] - A correspondence theorem for projective modules and the structure of simple Noetherian rings, Symposia Mathematica, vol. VIII (Convegno sulle Algebre Associative, INDAM, Roma, Novembre, 1970), Academic Press, London, 1972, pp. 309-345. MR 49 \#5088.

[73] - Algebra: Rings, modules and categories, Springer-Verlag, New York and Berlin, 1973.

[73] K. R. Goodeari, Subrings of idealizer rings, J. Algebra 33 (1975), 405-429.

[70] R. Hart and J. C. Robson, Simple rings and rings Morita equivalent to Ore domains, Proc. London Math. Soc. (3) 21 (1970), 232-242. MR 43 \#4857.

[43] N. Jacobson, The theory of rings, Math. Surveys, no. 2, Amer. Math. Soc., Providence, R. I., 1943. MR 5, 31. 
[64] J. P. Jans, Rings and homology, Holt, Reinhart and Winston, New York, 1964. MR 29 \#1243.

[71] A. V. Jategaonkar, Endomorphism rings of torsionless modules, Trans. Amer. Math. Soc. 161 (1971), 457-466. MR 44 \#1690.

[68] E. Matlis, Reflexive domains, J. Algebra 8 (1968), 1-33. MR 36 \#3765.

[74] B. J. Müller, The quotient category of a Morita context, J. Algebra 28 (1974), $389-407$.

[72] J. C. Robson, Idealizers and hereditary noetherian prime rings, J. Algebra 22 (1972), 45-81. MR 45 \#8687.

[67] J. M. Zelmanowitz, Endomorphism rings of torsionless modules, J. Algebra 5 (1967), 325-341. MR 34 \#2626.

DEPARTMENT OF MATHEMATICS AND PHYSICS, RIDER COLLEGE, BOX 6400, LAWRENCEVILLE, NEW JERSEY 08648 